Nouvelles perspectives en sciences sociales

\title{
Un paradigme systémique relationnel est-il possible? Proposition d'une typologie relationnelle
}

\section{Rachid Bagaoui}

Volume 3, numéro 1, septembre 2007

URI : https://id.erudit.org/iderudit/602468ar

DOI : https://doi.org/10.7202/602468ar

Aller au sommaire du numéro

\section{Éditeur(s)}

Prise de parole

ISSN

1712-8307 (imprimé)

1918-7475 (numérique)

Découvrir la revue

Citer cet article

Bagaoui, R. (2007). Un paradigme systémique relationnel est-il possible? Proposition d'une typologie relationnelle. Nouvelles perspectives en sciences sociales, 3(1), 151-175. https://doi.org/10.7202/602468ar
Résumé de l'article

Régulièrement les sociologues s'interrogent sur l'unification de leur savoir et sur les moyens d'y parvenir. Certains croient que non seulement l'atteinte de cet objectif est possible, mais qu'ils possèdent déjà un paradigme : l'analyse relationnelle. Paradoxalement, soutiennent-ils, la sociologie, jusqu'à maintenant, n'a pas su se donner ce paradigme comme principe unificateur. Nous défendons l'idée qu'il existe des sociologies relationnelles, comme le montre la typologie que nous proposons. De plus, que la relation ne peut, pour différentes raisons, servir de principe unificateur. Nous voyons en elle plutôt un terrain de confrontation entre les tenants de l'approche relationnelle et au sein de chaque approche se réclamant de l'analyse relationnelle. 


\title{
Un paradigme systémique relationnel est-il possible ? Proposition d'une typologie relationnelle
}

\author{
RACHID BAGAOU! \\ Université Laurentienne
}

À une question posée par La revue du MAUSS ${ }^{1}$, "Une théorie sociologique est-elle pensable ? ", la réponse de Pierpaolo Donati ${ }^{2}$ est sans équivoque. Non seulement elle est pensable, mais la tradition sociologique possède déjà un paradigme : il s'agit du caractère relationnel des phénomènes. Paradoxalement, la sociologie ne s'est pas donné ce paradigme comme terrain de confrontation. Talcott Parsons ${ }^{3}$, par exemple, à qui on doit la tentative la plus poussée d'unification de deux grandes traditions sociologiques «la théorie de l'action » et « la théorie des systèmes », a échoué précisément parce qu'il n’a pas su problématisé le lien entre le système d'action et les différents éléments ou soussystèmes et a négligé les relations en tant qu'effets émergents. Karl Marx, lui-même, que l'on peut ranger dans une sociologie relationnelle, n'a pas su faire de la relation le cœur de la démarche, car il est resté

Revue du MAUSS, Une théorie sociologique générale est-elle pensable ?, $\mathrm{n}^{\circ}$ 24, 2004.

Pierpaolo Donati, «La relation comme objet spécifique de la sociologie ", Revue du MAUSS, n० 24, 2004, p. 234-254.

Talcott Parsons, Théorie générale des systèmes, Paris, Dunod, 1973. 
enfermé dans une sociologie réaliste et a réduit toutes les dimensions de la vie sociales à l'aspect économique. On peut en dire autant du structuralisme, de la systémique et de bien d'autres. En fait, de manière générale, les sociologues, malgré leurs efforts, ne font qu'observer différents aspects de la relation : les symboles, les structures, les fonctions, les formes de communication, etc. et se disputent pour savoir quelle dimension peut être considérée comme facteur principal. Cela a conduit à l'éclatement de la sociologie en différents courants : la théorie de l'action, le fonctionnalisme systémique, la théorie critique et la théorie narrative où chacune tente de se disputer une dimension de la vie sociale.

Pour dépasser ces clivages, Donati propose de revenir à l'objet premier de la sociologie. Cet objet n'est ni le sujet, ni le système social, ni l'action, mais la relation. La relation possédant sa réalité propre, qui ne provient de rien d'autre. La relation comme système de référence. La relation comme point de vue premier à partir duquel la sociologie tente de saisir son objet. Une sociologie qui ne cherche pas à étudier la relation entre les réalités sociales, mais plutôt les réalités sociales en tant que relations sociales. C'est ainsi que l'on peut comprendre des phénomènes comme le don, l'État-providence ou la famille. Cependant, si l'unification de la sociologie autour de la relation est possible, cela ne peut être envisagé et structuré, selon l'auteur, qu'en accord avec l'analyse de réseau. Hors réseau, point de salut. L'analyse de réseau réussira là ou les autres ont échoué.

C'est aussi à cette tâche que Bajoit a consacré son livre Pour une sociologie relationnellet. 11 a cherché à sortir la sociologie de la crise qui s'exprime pour lui dans l'impossibilité de sortir des débats stériles entre la structure et l'action et ses multiples variantes. Comme Donati, Bajoit veut aussi dépasser l'éclatement des paradigmes en sociologie : le paradigme du « on », postulat de la théorie normative, le fonctionnalisme en général ; le paradigme du " moi », symbolisé par Weber, l'interactionnisme; le paradigme du " eux », que l'on retrouve présent chez les structuralistes ; et, enfin, le paradigme du « nous » que l'on retrouve principalement chez les théoriciens des mouvements sociaux et dans le marxisme. Pour intégrer et dépasser les quatre paradigmes, il 
propose aux sociologues de se doter d'un paradigme relationnel qui engloberait toutes les dimensions de la vie sociale, faute que de quoi elle risque de penser le $\mathrm{XXI}^{c}$ siècle avec des théories du XIX ${ }^{c}$ siècle. Mais de quel paradigme relationnel s'agit-il ? Tout ce qui peut être qualifié de relationnel ne peut venir que des relations entre individus et des ensembles d'individus : «Les conduites des individus sont sociales dans la mesure où elles portent les marques des relations qu'ils ont eues, qu'ils ont, ou qu'ils comptent avoir avec d'autres. La question que nous posons est de savoir de quoi est fait ce lien qui rend sociales les conduites des hommes, en faisant que chacun tienne compte des autres quand il agit ${ }^{5}$ ». Tous les efforts de synthèse le conduisent à privilégier une perspective particulière, celle du sujet. Pour s'en convaincre, examinons une des formes principales de la relation sociale qu'il propose : les échanges sociaux. Les liens d'échange regroupent quatre formes différentes : complémentaires, compétitives, conflictuelles et contradictoires. Or, et c'est le plus important, ces formes se caractérisent par la nature de leurs finalités et par les modes de reproduction de l'inégalité entre les sujets en relation. En fait, ces échanges sont simplement des stratégies par lesquelles les acteurs tentent d'exercer leur emprise sur les finalités de la relation et sur les modes de contrôle de l'inégalité. Une stratégie peut être définie comme "un ensemble cohérent de conduites, individuelles ou collectives, mises en œuvre par les membres d'une catégorie sociale, pour conserver ou pour s'assurer une meilleure emprise sur les finalités qu'ils poursuivent dans l'échange, en s'efforçant de maintenir ou d'améliorer leur position inégale dans la relation ${ }^{6} »$. On peut faire la même remarque en parlant de l'autre forme d'échange qu'est le lien de solidarité. Encore là, les acteurs, en s'identifiant à un groupe, poursuivent des finalités que l'auteur qualifie d'instrumentale : chacun a besoin des autres pour réaliser son intérêt.

Les propositions de Donati ou de Bajoit soulèvent des questions. Estil juste de soutenir que la sociologie a renoncé à toute tentative d'unification autour d'un paradigme relationnel ? Nous défendons, quant à nous, une position différente. Il existe bel et bien une sociologie relationnelle ou plutôt des sociologies relationnelles. Plusieurs auteurs

Ibid, p. 90.

Ibid, p. 98. 
se sont aventurés dans cette voie. Pensons à la théorie du don ${ }^{7}$, à la théorie des champs ${ }^{8}$, à la théorie de la configuration", à l'analyse des réseaux $^{10}$, à l'analyse stratégique ${ }^{11}$; à la théorie de la structuration ${ }^{12}$; à la théorie de l'échange ${ }^{13}$; au structuralisme ${ }^{14}$, etc. Ces approches prolongent, chacune à sa façon, une longue tradition philosophique constituée autour de la question relationnelle que l'on trouve chez des philosophes comme Pascal, Spinoza, Nietzche, Bergson, Tarde... mais aussi chez des fondateurs et des classiques de la sociologie, particulièrement chez Simmel, Marx et Weber.

Il faut reconnaître cependant qu'il est difficile de mettre de l'ordre dans cette pluralité d'analyses relationnelles, d'autant plus qu'il n'existe pas, à notre connaissance, une typologie relationnelle; les typologies qui existent mobilisent des catégories classiques comme l'intégration, le conflit, l'ordre, etc. Berthelot ${ }^{15}$, en se livrant à un exercice différent, a déjà attiré l'attention sur les difficultés qui se posent au chercheur lorsqu'il veut appréhender la pluralité des théories en sociologie. Il avoue même qu'aucun critère ne peut satisfaire cette exigence (même celui de programme utilisé par l'auteur). La question est donc de savoir comment appréhender cette pluralité d'analyses relationnelles.

Jacques T. Godbout et Alain Caillé, L'Esprit du don, Paris, La découverte, coll. « La découverte poche, Sciences humaines et sociales », 2000.

* Voir, entre autres, Pierre Bourdieu, Questions de sociologie?, Paris, Minuit, 1981.

" Voir, particulièrement, Norbert Elias, Qu'est-ce que la sociologit, Paris, Fayard, 1970, et La saciéte des individus, Paris, Fayard, 1991.

11) Voir, entre autres, Emmanuel Lazega, Réseaux sociaux et structures relationnelles, Paris, PUF, coll. "Que sais-je ? », 1998 ; Stanley Wasserman et Katherine Faust, Social network analysis: Methods and applications, N ew York, Cambridge University Press, 1994 ; Alain Degenne et Michel Forsé, Les réseaux sociaux, Paris, Armand Colin, 1994.

11 Voir Michel Crozier et Erhard Friedberg, L'Acteur et le système, Paris, Seuil, 1977.

Anthony Giddens, La constitution de la société, Paris, PUF, 1988.

1.3 Marcel Mauss, Sociologie et anthropologie, Paris, PUF, coll. "Sociologie aujourd'hui ", 1973.

14 Claude Lévi-Strauss, Les structures élémentaires de la parenté, Paris, Mouton, Maison des sciences de l'homme, coll. "De rééditions ", 1967 et Antbropologie structurale, Paris, Plon, 1958.

15 Jean-Michel Berthelot, L'intelligence du social. Le pluralisme explicatif en sociologie, Paris, PUF, coll. «Sociologie d'aujourd'hui », 1997. 
De plus, on peut se demander en quoi les perspectives défendues par ces auteurs sont plus relationnelles que d'autres ${ }^{16}$ ? L'analyse des réseaux ou le paradigme relationnel de Bajoit représentent, il est vrai, une tentative louable d'unification de la sociologie autour d'un seul paradigme. Nous sommes cependant sceptiques face à un tel exercice. Nous considérons la notion de relation, au même titre que les autres catégories (configuration, système, champs, réseaux...), comme un terrain de confrontation, et non comme un principe unificateur. Nous ne voulons pas laisser entendre par notre propos que toute tentative d'unification autour de la notion de relation est inutile : elle a au moins le mérite de faire avancer le débat sur le statut des théories sociologiques. Nous voulons dire que les auteurs oublient que revendiquer un paradigme unificateur, c'est nécessairement marquer une différence. Cette différenciation crée d'autres positionnements et, du coup, cela rend l'unification impossible. Au lieu de demander à la notion de relation de jouer un rôle unificateur, nous lui demandons de servir de terrain de confrontation entre les tenants de l'approche relationnelle et au sein de chaque approche se réclamant de l'analyse relationnelle.

Notre objectif est plus modeste que celui de Berthelot. Il est de fournir un instrument pédagogique qui permet de voir clair dans les nombreuses utilisations de l'analyse relationnelle en différentes lorgnettes et à l'intérieur de chacune d'elles. Une attention particulière sera accordée à la pensée systémique qui demeure, à notre avis, la tentative la plus sérieuse de fonder une sociologie relationnelle interdisciplinaire. Nous ne cherchons pas à proposer une solution pour dépasser les clivages entre les perspectives. Nous ne discuterons pas de manière approfondie les auteurs que nous jugeons représentatifs des approches retenues. Il existe pour cela des manuels très sérieux. De plus, le choix des auteurs que nous faisons est forcément limité. Le lecteur convaincu par notre typologie pourra toujours en discuter d'autres à la lumière de cette typologie et ajouter lui-même ceux qu'il juge conformes à certains types. Il est vrai, qu'une typologie, en fin de compte, participe à sa manière à la perpétuation des catégories au nom desquelles

On peut formuler la même question à propos de l'article de Mustafa Emirbayer qui se veut proche de l'analyse transactionelle (voir " Manifesto for a relational sociology ", American joumal of sociology, vol. 103, $\mathrm{n}^{\circ}$ 2, septembre, p. 281-317). 
s'affrontent les sociologues, mais sans qu'elle ait pour finalité tel objectif. Nous espérons, cependant, que cette cartographie simplifiée d'une sociologie relationnelle servira un jour, qui sait, comme outil pour jeter un pont entre les perspectives et les auteurs.

\section{Vers une typologie relationnelle}

Nous évitons de partir d'une définition de la relation. Elle sera dégagée à même les contributions des auteurs. En nous appuyant sur plusieurs auteurs (voir la bibliographie), il est possible de proposer une typologie relationnelle sur un axe comptant trois niveaux. Tous les auteurs qui leur sont attachés n'ont pas forcément discuté la relation de manière explicite, mais tous ont en commun d'avoir développé une sociologie relationnelle. De plus, toutes ces perspectives cherchent à résoudre, dans une optique relationnelle, la dichotomie principale en sociologie entre individu et société, et ses variantes.

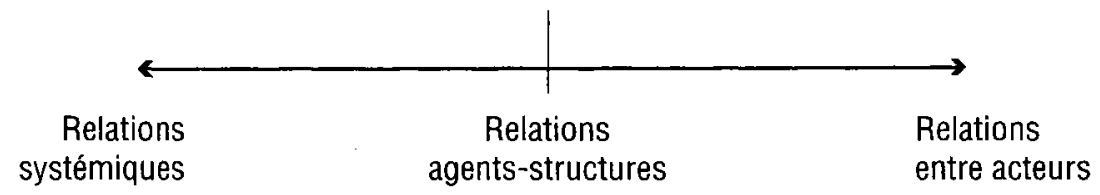

\section{Relations entre acteurs}

Le premier cas de figure relationnel, situé à droite de l'axe, est qualifié de relations entre acteurs (effets des interactions). Nous pouvons regrouper des auteurs qui se positionnent dans la théorie de la configuration, l'analyse de réseaux, l'analyse stratégique, les analyses des mouvements sociaux, le marxisme, l'interactionnistes ${ }^{17}$. Tous ces auteurs partent de l'idée que la totalité n'existe pas en soi. Il n'y a pas de boîte noire. Il n'y a que des rapports entre individus et des ensembles d'individus. Dans tous les cas, la réalité est un construit social. Ce sont les acteurs à travers

Par exemple, Erving Goffman (Asiles : etudes sur la condition sociale des malades mentaux et autres reclus, Paris, Minuit, 1968 ; La mise en scène de la vie quotidienne, Paris, Minuit, 1973 ; Les rites d'interaction, Paris, Minuit, 1974). 
leurs luttes, leurs alliances, leurs perceptions... qui construisent le monde. La structure n'est pas évacuée pour autant. On essaye de comprendre comment la structure contraint les comportements tout en faisant émerger les interactions. Mais cette structure n'est pas extérieure aux relations entre individus. Elle est le produit des relations sociales.

Cependant il existe des différences importantes au sein de cet ensemble. Dans la sous-perspective effets d'émergence, nous rangeons Elias et les défenseurs de l'analyse du réseau. Crozier et les interactionnistes sont associés à la sous-perspective jeux entre acteurs. Enfin, les marxistes, les théoriciens des mouvements sociaux appartiennent la sousperspective enjeux entre acteurs.

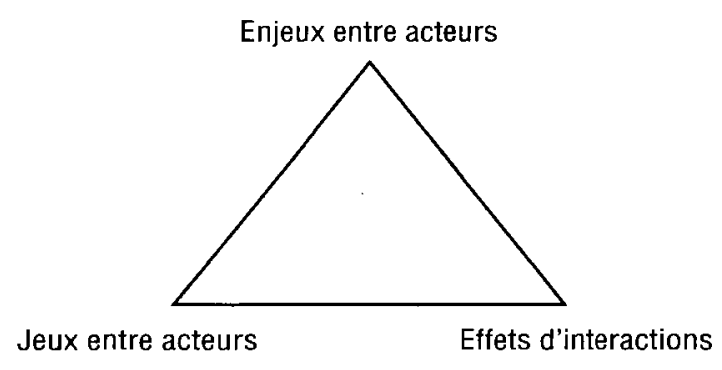

La sous-perspective effets d'interactions perçoit le social comme étant des interactions interdépendantes entre les individus ou encore entre des groupes à divers niveaux. Les classes sociales, la famille, l'État ou le système de santé, par exemple, ne sont pas extérieurs à l'individu, mais le produit non voulu des interactions entre acteurs. Chez Elias, cela s'exprime par la notion de configuration. Elle se définit comme une formation de taille variable (les joueurs d'une partie de cartes, la société d'un café, une classe scolaire, un village, une ville, une nation) où les individus sont liés les uns aux autres par un mode spécifique de dépendances réciproques et dont la reproduction suppose un équilibre mobile de tensions. Les défenseurs de l'analyse de réseaux s'orientent dans la même direction. Les catégories sociales (classe, groupe, mouvement, etc.), définies par des caractéristiques sociodémographiques, ne sont que le reflet des relations structurales qui lient les individus. Au lieu de partir d'agrégats définis a priori par les caractéristiques sociodémographiques que tous les membres possèdent 
en commun, on prend en considération l'ensemble des relations dans une population donnée. L'analyse de réseaux tente de trouver les régularités de comportements et les groupes qui les génèrent (fréquence des liens, échanges, etc.) de manière inductive. La structure du réseau est considérée comme effet émergent, car les relations qui s'y nouent vont affecter cette structure.

Crozier exprime bien la sous-perspective jeux entre acteurs. Malgré son appel à la systémique, ce qui compte, pour lui, principalement, c'est le jeu entre acteurs. Pour Crozier, le système est un construit humain. L'organisation, objet d'étude de l'auteur, est composée d'acteurs structurant leurs relations dans un modèle aussi interactif qu'interdépendant. La manière dont cet ensemble sera construit sera nommée système d'action concret. Il s'agit de la manière dont les acteurs construisent le système de relations pour résoudre les problèmes concrets de l'organisation. Ils le font suivant en cela leur propres visées, qui sont des compromis entre leurs propres objectifs et ceux de l'organisation.

Enfin, chez les analystes des mouvements sociaux (Touraine par exemple ${ }^{18}$ ) ou chez les marxistes, représentants de la sous-perspective enjeux entre acteurs, les relations n'ont de sens que par rapport à l'enjeu de dispute. Que ce soit pour le contrôle de l'historicité, pour l'appropriation des moyens de production ou pour d'autres objectifs, l'enjeu est posé d'avance et les acteurs entrent en conflit pour s'approprier les termes de la dispute.

\section{Relation agents-structure}

Le deuxième cas de figure, situé au milieu de l'axe, est la relation agentsstructures. Nous pensons ici à Bourdieu, Archer, Giddens, Alexander ${ }^{19}$. Il s'agit de résoudre de manière relationnelle la question de l'agent et de la structure. Dans cette perspective, les agents et leurs relations existent et ont un poids aussi important que la structure. Ces auteurs offrent,

18 Nous parlons du deuxième Alain Touraine, l'observateur des mouvements sociaux. Voir La voix et le regard (Paris, Seuil, 1978) ou Pour la sociologie (Paris, Seuil, 1974).

19 Jeffrey Alexander, Theorical logic in sociology, vol. 1-4, Berkley, University of California Press, 1982-1983. 
selon notre typologie, trois manières d'exprimer la relation entre les deux aspects de la vie sociale.

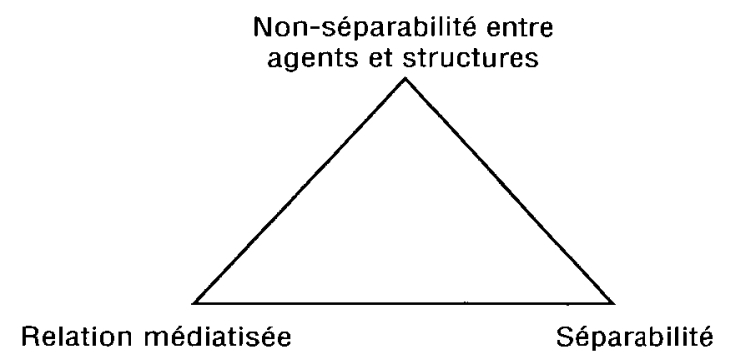

Chez Giddens, il n'y a pas de séparation entre structures et agents, mais co-constitution. L'étude de la structuration des systèmes sociaux est celle des modes par lesquels les systèmes s'ancrent dans des actions d'individus, doués de compétences. Ces derniers ont des raisons de faire ce qu'ils font et sont parfois capables d'exprimer ces raisons de façon discursive, parfois non. Ils utilisent des ressources et des règles dans une diversité de contextes d'actions, contextes qui sont produits par l'interaction des acteurs et qui produisent eux-mêmes ces acteurs. La structure et l'agent ne sont pas deux aspects indépendants. Les propriétés structurelles sont à la fois le moyen et le résultat des pratiquent qu'elles organisent de façon discursive. Chez Bourdieu, il y a une relation entre les positions, car l'acteur est défini par une position relative à celle de tous les autres acteurs, en termes de similarités ou non, aux plans économique, culturel, social et symbolique; mais cette relation n'est pas concrète ou directe. La relation existe aussi entre les positions et le champ ; cependant, ces deux aspects ne sont pas fusionnés, comme chez Giddens, mais médiatisés par l'habitus ${ }^{20}$. L'accord entre les deux est certes la règle générale, mais il existe aussi des moments de crises qui

24. Cette position n'est pas partagée par Jeffrey Alexander qui ne croit pas que ce concept désigne un lien entre agent et structure, ou, ce qui revient au même, entre micro et macro, puisqu'il ne correspondrait finalement à rien d'autre qu'à la forme empirique d'une position de classe (voir son livre La réduction. Critique de Bourdieu, Paris, Cerf, coll. « Humanités », 2000). Telle n'est pas notre opinion. 
révèlent la possibilité d'un désajustement entre les deux aspects. Chez Archer $^{21}$, par contre, il n'y a ni lien direct ni médiatisation, mais séparation entre l'agent et la structure. Même s'il y a une relation intime entre les deux aspects de la vie sociale, ils sont analytiquement distincts. Trois moments à distinguer : le conditionnement structurel, les interactions et l'élaboration structurale. Chacun de ces aspects possède ses propriétés émergentes autonomes qui ne se trouveront pas en phase les unes avec les autres. La structure est nécessairement antérieure aux actions qui la transforment ; l'élaboration de la structure est nécessairement postérieure à ces actions.

\section{La pensée systémique}

Pour la troisième perspective, la pensée systémique ${ }^{22}$, l'accent est mis sur la totalité, définie comme l'ensemble des relations entre les parties. Mais ce qui se passe du point de vue des échanges entre les parties est plus que l'expression des qualités des parties. Cette totalité, qualifiée de

21 Margaret Archer, "Entre la structure et l'action, le temps. Défense du dualisme analytique et la perspective morphogénétique ", Revue da MAUSS, n ${ }^{\circ} 24,2004$, p. 237-349 ; Realist social theory: The morphogenetic approach, Cambridge, Cambridge University Press, 1995.

22 Ludwing Von Bertalanffy, Théorie générale des systèmes, Paris, Dunod, 1990 ; Daniel Durand, La systémique, Paris, PUF, coll. «Que sais-je ? ", 1993 ; Edgar Morin, Introduction à la pensée complexe, Paris, ESF, 1992 ; Le défi dit XXI siècle. Relier les connaissances, Paris, Seuil, 1999 ; La tête bien faite. Repenser la réforme. Réformer la pensée, Paris, Seuil, 1999 ; Edgar Morin et Jean-Louis Le Moigne, L'intelligence de la complexité, Paris, L'Harmattan, 1999 ; Joël de Rosnay, Le macroscope. Vers une vision globale, Paris, Seuil, coll. « Points ", 1975 ; Yves Barel, Le paradoxe et le système, Presses universitaires de Grenoble, 1989 ; Jean-William Lapierre, L'analyse des systèmes, Syros, Paris, 1992 ; Jean-Claude Lugan, La systémique sociale, Paris, PUF, coll. "Que-sais-je ?», 1995 ; Lexique systémique et prospective, Toulouse, Conseil économique et social de la région Midi-Pyrénées, 2006 ; Niklas Juhmann, « Développements récents en théorie des système ", dans Gérard Duprat, Connaissance du politique, Paris, PUF, 1990 ; Excological communication, Chicago, University of Chicago Press, 1989 ; Social systems, Standford, Stanford University Press, 1995 ; Simon Laflamme, Des biens, des idées et des personnes au Canada (1981-1995): un modele macrosociologique relationnel, Sudbury/Paris, Prise de parole / I.'Harmattan, $2000 ;$ La société intégrée. De la circulation des biens et des idées, New York / Berne, Peter Lang, Worcester Polytechnic Institute, Studies in Science, Technology and Culture, 1992 ; Paul Watzlawick, Janet H. Beavin et Don D. Jackson, Une logique de la communication, Paris, Seuil, 1972. 
système pour la plupart des auteurs (sauf chez Laflamme) se présente comme un ensemble de relations formant une unité organisée, ayant sa propre identité et se distinguant de son environnement. Ce qui compte ici, c'est comment les parties sont organisées. Il n'y a donc pas, d'un côté, les parties et, de l'autre, le tout. Comme les autres perspectives, la systémique aussi est une pensée relationnelle plurielle. Dans les pages qui suivent, nous allons examiner plus en détails cette perspective et sa pluralité.

\subsection{L’analyse relationnelle systémique}

Rappelons que la systémique découle de différentes sources, qu'il serait impossible d'énumérer dans le cadre de ce court texte : la logique formelle (Bertrand Russel et le Théorème de Gödel), la théorie des systèmes, la théorie de l'ordre et du bruit, la théorie du désordre, etc. Elle concerne la biologie, la sociologie, la philosophie, la physique et bien d'autres disciplines. La systémique se veut une réaction à plusieurs tendances. Contre le réductionnisme, elle veut rendre compte de la complexité grandissante du social. Contre le relativisme scientifique, elle prône l'application presque universelle de la méthode scientifique. Contre l'isolement et la dispersion du savoir, elle regarde à travers des totalités de plus en plus vastes. Contre l'éclatement, elle cherche des concepts, des lois et des modèles dans différents domaines de la connaissance. Contre l'académisme, elle prône la nécessité de développer des modèles de résolution de problème transférables dans les conditions du monde réel. Contre le déterminisme excessif, le réel est lu comme indéterminé, contingent et anarchique. Le «bruit» demeure nécessaire à la création des ordres nouveaux. Contre la certitude, le paradoxe est vu comme inhérent à la réalité physique du monde naturel et psychique du sujet.

La systémique repose sur l'hypothèse qu'un ensemble possède des propriétés émergentes et qu'il est donc impossible d'atteindre la compréhension de ce tout par l'étude exclusive de ses parties qui le constituent. Bertalanffy confirme cette position, dès le début des années 1970 , en soulignant que cette tendance est compatible avec la science contemporaine qui veut « ne plus isoler les phénomènes dans des contextes étroitement confinés, [...] ne plus décortiquer les interactions avant de les examiner, $[. .$.$] regarder des "tranches de nature" de plus en$ 
plus larges ${ }^{23} »$. Watzlawick et ses collaborateurs expriment tôt cette idée de la façon suivante : «Un phénomène demeure incompréhensible tant que le champ d'observation n'est pas suffisamment large pour qu'y soit inclus le contexte dans lequel ledit phénomène se produit ${ }^{24}$ ». Cette tendance est souvent traduite par l'expression « le tout est plus que la somme des parties » et selon laquelle on ne peut les connaitre vraiment sans les considérer dans leur ensemble. Cette globalité exprime à la fois l'interdépendance des éléments du système et la cohérence de l'ensemble. Sous le nom d'approche globale, le concept désigne également la voie d'entrée dans la démarche systémique : partir d'une vue générale (globale) pour approfondir les détails, avec de nombreuses itérations et des retours en arrière pour compléter ou corriger la vision antérieure. La pensée systémique repose également sur une hypothèse complémentaire à savoir que la connaissance de l'objet doit passer par l'étude des relations et des interactions qu'a cet objet ou cet ensemble avec son environnement. Il en est ainsi puisque environnement et système s'influencent mutuellement. Ce qui est suggéré ici, c'est l'opposé du précepte réductionniste qui préconise la décomposition, la réduction et l'isolement, de l'objet ou du phénomène, de son environnement pour mieux l'étudier.

Le socle sur lequel repose la systémique est sans doute la notion de système. Il serait vain d'en énumérer toutes les définitions ${ }^{25}$. Toutes les définitions ont pour dénominateur commun l'existence, au sein d'un système, d'un ensemble d'éléments interreliés. Les autres aspects qui sont associés à la notion de système n'apparaissent pas automatiquement $^{26}$. Limitons-nous à l'essentiel. Un système est une combinaison d'éléments dont la réunion forme un ensemble. L'articulation de ces éléments en réseaux délimite le système, lui donne son autonomie, sa spécificité, le différencie. C'est en cela que nous disons que la

2.3 I.udwing von Bartalanffy, op. cit., p. 8.

24 Paul Watzlawick et al., op. cit., p. 15.

25 Voir les différentes définitions proposées dans Jean-Claude Iugan (dir.), Lexique systémique et prospective, op. cit., p. $81-82$.

26. Il existe plusieurs ouvrages et articles qui traitent de la systémique. Les points qui reviennent le plus souvent sont la relation entre les éléments, la totalité, l'organisation, la complexité et les régulations. 
systémique est une pensée relationnelle. En effet, les éléments, tout d'abord, n'existent pas en eux-mêmes. C'est leur interaction qui compte. En systémique, on ne considère pas tour à tour les éléments et, par après, leurs interactions ; au contraire on s'intéresse au produit de l'interaction des éléments, produit impossible à prendre en compte par les stratégies traditionnelles de division et de simplification. Ensuite, même si elles peuvent procéder du chaos, les interactions ne sont pas du chaos, elles s'organisent en un tout et n'ont de sens que par rapport à ce tout qui n'est pas réductible aux parties. Enfin, poser ce tout en tant qu'organisé en système, c'est penser relationnellement, non seulement parce que c'est se donner un ensemble de relations internes, mais aussi parce que cet ensemble n'existe que par rapport à un autre tout organisé. Le système entretient des relations avec son environnement. Il en résulte des changements plus ou moins contrôlés que subissent les intrants provenant d'autres systèmes. Ces transformations produisent à leur tour des extrants qu'absorbe l'environnement. Cependant, ces transformations se feront en fonction des finalités du système. Ces critères permettant de vérifier si le système est en situation d'échec ou de réussite.

$\mathrm{Si}$, pour comprendre le caractère relationnel de la systémique, les concepts de globalité et d'interaction sont fondamentaux, il y en a aussi d'autres dont le rôle est important. Nous pensons aux concepts d'information, de rétroaction, de finalité, d'ago-antagonisme, de causalité circulaire, de régulation, d'organisation, de variété, etc. ${ }^{27}$ Tous ces concepts ont une même fin analytique : s'attacher plutôt à l'unité d'un système donné qu'à ses composants, ou encore davantage à son comportement comme tout non dissociable qu'aux caractéristiques de ses composants.

\subsection{Une pensée relationnelle diversifiée}

Comme le notent les systémiciens, avec la pensée systémique et ses concepts, les différentes disciplines sont ainsi outillées pour commencer à se parler et à développer une lecture homogène de leur travail. Si ce

27 Pour en connaitre plus sur l'outillage de la systémique, le lecteur peut consulter JeanClaude Lugan (dir.), Lexique systémique et prospective, op. cit. 
but demeure louable, nous croyons que, pour le moment, la pensée systémique est plutôt marquée par la pluralité. Les tenants de la pensée systémique ont trop tendance à oublier cette réalité, ce qui réduit considérablement l'espace de confrontation au sein de cette perspective. Pour souligner cette pluralité, nous avons identifié trois manières de penser relationnellement au sein de la systémique : la perspective échangiste; la perspective différentialiste (ou système-environnement); et la complexité systémique ou relations toalité-parties.

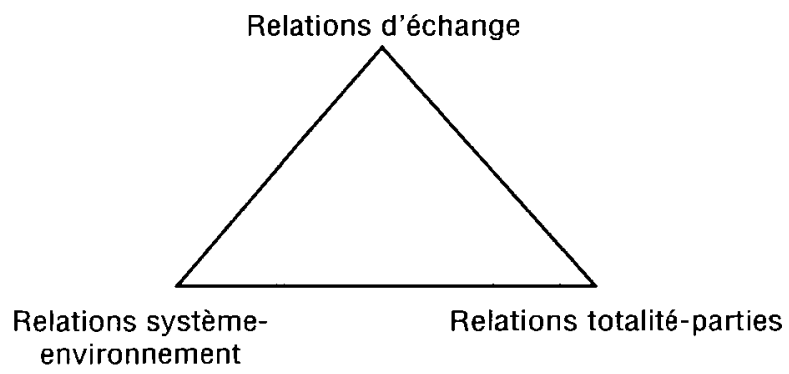

On peut illustrer l'échange systémique (relations d'échange) par la contribution de Simon Laflamme. La différenciation systémique (système/environnement), quant à elle, peut avoir pour exemple le travail de Niklas Luhmann et la complexité systémique (relations des parties au tout), l'œuvre d'Edgar Morin.

\subsubsection{La différenciation systémique}

La différenciation systémique est une illustration parfaite de ce souci de fonder une sociologie relationnelle. Chez Luhmann, la société ne se compose pas d'individus. Un être humain n'est pas un système. Il est litigieux de chercher à obtenir un système en rassemblant à l'intérieur d'un tout plusieurs êtres humains. Plus précisément, la société et les sous-systèmes ne se composent pas d'individus, mais de communications. Cela ne veut pas dire que la société puisse exister sans individus. Cela veut tout simplement dire que les individus ne font pas partie de l'autoréférentialité du système mais plutôt qu'ils en constituent 
l'environnement. Ils n'existent que sous forme de réseau ou d'ensemble d'attentes. De plus, l'analyse différentialiste ne part d'aucune catégorie a priori, comme l'intérêt, les motivations, le pouvoir ou les stratégies. La nature du réel s'exprime à travers les problèmes, au demeurant impossibles à éliminer. Dans cette perspective systémique, le hasard est central. Il contribue à transformer la complexité (ensemble des événements) en monde réel. Ce monde de possibilités, qui se nomme complexité, est un chaos, et non encore un cosmos. Lorsque la complexité n'est pas réduite, la société n'existe pas encore, et le problème prend la forme de la contingence. Par contingence, il faut comprendre ce qui n'est ni nécessaire, ni impossible, mais qui est simplement possible. Par contre, lorsque l'ordre est introduit, la complexité est réduite, et la société peut alors commencer. La réduction de la complexité est donc présente au commencement de tout ordre, à l'origine de toute interaction sociale ; et, de ce fait, elle constitue le moteur de l'évolution des systèmes sociaux. C'est grâce à ce travail, rappelons-le, qu'une société peut évoluer, devenir plus complexe.

Pour Luhmann, un système ne se définit donc pas comme un tout composé d'objets, de parties ou de relations; il se révèle comme une unité qui peut être distinguée de son environnement. Le plus important n'est pas la relation entre les parties et le tout, mais le système comme différenciation. Plus précisément, le système est une distinction systèmeenvironnement. Si le système est une distinction, il peut observer d'autres distinctions qui sont, elles aussi, de type systèmeenvironnement, comme lui, et il peut s'observer lui-même en tant que distinction système-environnement. La constitution des systèmes trouve son origine dans l'environnement. Mais, une fois autonomes, les systèmes alimentent, eux-mêmes, leur fonctionnement (phénomène d'autoréférence ou d'autopoïesis). Les systèmes autoréférents sont des systèmes qui produisent leurs éléments au moyen de leurs propres éléments ; mais ils sont aussi ce qui permet d'identifier le système comme unité (opérations, processus, structures). En tant qu'unité, le système n'est ni un contenu, ni une structure, ni une configuration stable des éléments. L'unité, c'est son mode de reproduction. Ainsi, l'autonomie du système, c'est l'autonomie de sa reproduction. Le système juridique, par exemple, pour Luhmann, ce ne sont ni les attentes, ni la totalité des actes juridiques qui ont lieu, ni une hiérarchie formelle; c'est la manière selon laquelle le droit peut se créer 
uniquement à partir du droit lui-même. C'est pourquoi la clôture du système représente la condition de son ouverture. Car s'il est en relation avec son environnement, c'est précisément qu'il se réfère à lui-même. Clôture ne signifie pas indépendance totale, mais autorégulation, par le système lui-même, de ses dépendances et indépendances vis-à-vis de l'environnement.

Dans chaque système il existe une règle propre et exclusive. Les systèmes se construisent et se maintiennent par le moyen de ce que Luhmann appelle le sens. Les limites des systèmes deviennent donc des limites de sens. Mais ce sens est un schéma formel et non un contenu, car il n'y a pas de substrat ontologique du sens. Il s'agit en fait d'un schéma binaire en termes d'appartenance ou de non appartenance. Dans le cas du système juridique, par exemple, le système fonctionne selon un code binaire, légal / non légal, qui détermine ce qui tombe sous le sens du juridique et de ce qui reste en marge du juridique (science, morale, etc.). Les éléments filtrés qui feront partie du système forment une structure et cette structuration seule permettra la permanente reproduction du système et la reproduction ordonnée des éléments dans le système. Ces éléments, on se le rappellera, sont des communications. Les structures de chaque système rendent prévisibles les communications qui se déroulent les unes par rapport aux autres dans le système. C'est ainsi que les systèmes peuvent se stabiliser et accomplir leurs fonctions.

Il est à peine besoin de mentionner le caractère relationnel de cette perspective. Poser l'idée de système comme autoréférent, c'est nécessairement s'inscrire dans une perspective relationnelle; car un système, comme nous l'avons mentionné, n'existe que par rapport à l'environnement. Sa particularité, qu'il convient de noter encore une fois, ne se définit ni comme un tout comportant des parties ni comme une relation entre le tout et les parties, et encore moins par référence à une finalité quelconque.

\subsubsection{La complexité systémique}

La complexité systémique de Morin représente une autre variante de l'analyse relationnelle systémique. La particularité d'Edgar Morin est d'avoir fait du système une notion visant à rassembler et à unifier des savoirs jusque là éclatés. Morin pousse à l'extrême les acquis 
systémiques en cherchant à fonder une perspective et une méthode générales qui unifieraient toutes les disciplines scientifiques, des sciences de la terre aux sciences sociales. Sa conception est moins différencialiste qu'homogénéisante. Les sciences doivent avoir pour objet non pas des dimensions, secteurs, de la vie sociale ou naturelle, mais un système complexe formant un tout organisateur et organisé. Comme celle de Luhmann, la pensée de Morin est une pensée du paradoxe. Que ce soit dans L'bomme et la mort ${ }^{28}$, Le cinéma ou l'bomme imaginaire ${ }^{29}$ et dans bien d'autres ouvrages, le paradoxe n'est pas une anomalie ; il fait partie du social. En plus d'être multidimensionnelle, la vie est aussi traversée par des paradoxes et des dichotomies : le réel et l'imaginaire ; la mort et la vie ; mais, également, de manière plus générale, le cultuel et le biologique ; l'individu et la société ; le sujet et l'objet ; la raison et la folie ; l'ordre et le désordre, etc. Ces paradoxes, ces dichotomies constituent en même temps la complexité. Mais cette complexité n'a rien à voir avec celle de Luhmann. Chez ce dernier, il est la tentative du système de réduire la complexité, donc de se différencier, de limiter ses frontières et de se reproduire. Pour Morin, penser la complexité, c'est penser les interactions entre le tout et les parties - la société et les individus -, c'est penser les phénomènes d'émergence, d'autoorganisation, les événements singuliers... en pensant en même temps l'enchevêtrement des divers facteurs explicatifs qui renvoient euxmêmes à différentes connaissances. Plus qu'un simple modèle, Morin propose une réforme de la pensée ${ }^{30}$ que Aït Abdelmalek résume comme " l'art d'organiser sa pensée, de relier et distinguer à la fois. Il s'agit de favoriser l'aptitude naturelle de l'esprit humain à contextualiser et à globaliser, c'est-à-dire à inscrire toute information ou toute connaissance dans son contexte et son ensemble ${ }^{31} »$.

Un système, selon Morin, est une unité globale organisée d'interactions entre éléments, actions ou individus. Le degré de complexité d'un système dépend du nombre de ses composants et du nombre et du type de relations qui les lient entre eux. Morin refuse la

2* Edgar Morin, L'bomme et la mort, Paris, Buchet/Chastel, 1951.

29) Edgar Morin, Le cinéna ou l'bomme imaginaire, Paris, Minuit, 1985.

3" Edgar Morin, La tête bien faite. Repenser la réforme. Réformer la pensée, Paris, Seuil, 1999.

31 Ibid., p. 13. 
dichotomie holisme-parties. Il faut abandonner ce schéma pour une explication circulaire où l'on va des parties au tout, du tout aux parties pour essayer de comprendre un phénomène. «La vision simplifiée serait de dire : la partie est dans le tout. La vision complexe dit : non seulement la partie est dans le tout; le tout est à l'intérieur de la partie qui est à l'intérieur du tout ! $!^{32}$ ». Mais il y a plus que ce principe pascalien, il y a aussi le principe de l'hologramme, qui est l'image physique dont les qualités de relief, de couleur et de présence tiennent au fait que chacun des points contient presque toute l'information de l'ensemble qu'il représente. On peut dire que toute la société est présente dans l'individu. La société comme système n'est pas intégrée et stable. Elle est traversée par des forces qui s'assemblent et s'apposent ; où ordre et désordre se mêlent; où les actions et les événements sont à la fois produits et producteurs de la réalité sociale ; où les bifurcations, les imprévus, les phénomènes d'auto-organisation, d'émergence... déjouent la régularité de l'ordre social.

L'analyse relationnelle de Morin, en accordant leur importance à la totalité et aux interactions entre les parties et cette totalité mobilise des concepts qui ont fait leur preuve dans d'autres disciplines : autoorganisation, récursivité, dialogie, etc. Comme les systémiciens en général, Morin refuse de voir dans quelque facteur que ce soit l'explication ultime. Il suffit de relire son livre Penser l'Europe $e^{3.3}$ pour s'en laisser convaincre. Les facteurs culturels, économiques, politiques, les multitudes d'influences, les différentes actions et les acteurs... dépendent les uns des autres et se combinent selon le principe de récursivité, c'est-à-dire que chaque facteur est à la fois produit et producteur des autres, qui se cristallisent en une forme auto-organisée.

\subsubsection{L'échange systémique}

S'il n'est pas toujours aisé d'établir des liens forts entre les perspectives luhmanienne et morinienne, celle de Laflamme tranche encore d'avantage avec elles. Laflamme ne parle ni de sujet ni de système, et il n'adopte pas toujours le langage systémique. Pourtant bien de points le

Edgar Morin, Introduction à la pensée complexe, Paris, ESF, 1992 p. 17.

3.3 Edgar Morin, Penser l'Furope, Paris, Gallimard, 1987. 
rapprochent de la perspective systémique. Pour la systémique échangiste de Laflamme, la relation est première, et non le système. La relation est un système d'échanges entre catégories abstraites : les idées, les biens et les personnes. Mais ces catégories en soi sont peu utiles pour l'étude. C'est leur lien qui compte. Il s'agit d'une dialectique. Les idées font circuler des personnes liées à des biens. Les biens font circuler des idées liées à des personnes. Les personnes font circuler des idées liées à des biens $^{34}$. Notons aussi que ces catégories d'échange ne se réduisent ni à des groupes concrets ni à des catégories comme l'intentionnalité, l'intérêt, le pouvoir, les classes sociales. Chez cet auteur, en effet, l'observateur ne peut comprendre le social s'il se place du point de vue des acteurs. L'acteur participe, certes, à l'échange mais « il ne se comprend pas lui-même dans une logique échangiste, parce que son action, telle qu'il peut la concevoir, relève d'une conscience inhérente au phénomène et, donc, n'a rien à voir avec l'interprétation que peut en faire le théoricien ${ }^{35} »$. La compréhension de l'acteur peut être prise comme objet d'étude, mais non comme un principe explicatif de la réalité. Sa lecture du monde n'obéit pas aux mêmes contraintes langagières : logique interne, mécanisme démonstratif, économie de concepts, articulation aux théories antérieures, etc.

Cet échange entre catégories cependant n'est pas un simple jeu entre éléments, comme on peut le constater chez des interactionnistes par exemple. Il est de nature systémique. Il se présente sous une forme intégrée, c'est-à-dire que si le social est divisé, éclaté, «il ne peut l'être que dans une forme particulière d'intégration de la circulation des composantes que retient le modèle $e^{36} \gg$. Peu de place est accordée ici au hasard, au chaos, dans le modèle d'analyse. La société est un système intégré. Et c'est cette intégration qui confère à la société son caractère spécifique et limite ses frontières. En même temps, la société entretient des relations avec les autres et, dans cette relation d'échanges, les sociétés forment un tout. Chez Laflamme, elle s'exprime parfois par "société postmoderne ", " société masse-médiatisée ", "société

\footnotetext{
34 Simon Laflamme, Des biens, des idées et des personnes an Canada (1981-1995): un modele macrosociologique relationnel, op. cit., p. 21.

35 Ibid., p. 14

3 Ibid., p. 15.
} 
intégrée », autant de termes qui renvoient à la dialectique entre "différenciation et homogénéités ${ }^{37}$ ».

\section{Interprétation}

Trois perspectives, trois manières de faire l'analyse relationnelle. Si, d'un côté, la systémique accorde de l'importance à la totalité et à la relation entre totalités pour comprendre la vie sociale, de l'autre, cette totalité n'existe pas ou, si l'on veut, elle n'est rien d'autre que des relations entre des acteurs. Au milieu de ces deux perspectives, il existe une troisième qui se donne pour objet de compréhension la relation entre l'agent et la structure. Chacune à sa façon, elles contribuent à donner une coloration particulière à la pensée relationnelle. Il est possible, à partir de là, de s'interroger de manière approfondie sur les passerelles à jeter entre ces perspectives. Cet objectif dépasse pour le moment le but de cet article. Nous croyons que cette typologie livre les premiers éléments pour un tel travail. Nous ne concevons pas, cependant, ces approches comme des blocs homogènes. Il faut qu'il y ait des débats au sein de chacune d'elles pour que la connaissance puisse se renouveler. Nous avons identifié trois sensibilités relationnelles au sein de chacune d'elles. Il devient alors possible de débattre autour de la notion de relation sans nécessairement adopter la même lecture à propos du statut du sujet, du rôle de la science, etc.

La perspective systémique illustre bien notre propos. Les défenseurs de cette perspective ont tous en commun le souci de fonder un savoir unifié, cherchant à intégrer non seulement les acquis de différentes disciplines, mais aussi différentes dimensions du social. Cela s'exprime par une théorie générale (le cas de Luhmann), un modèle (le cas de Laflamme) ou une méthode (le cas de Morin). Les trois cherchent à fonder une perspective relationnelle, mais chacune " la tient par un bout ". Le primat est accordé soit aux unités (Luhmann), soit aux catégories (Laflamme), soit aux parties et au tout (Morin).

De plus, deux éléments les distinguent : la place de l'acteur et le souci d'opérationnalisation de l'outillage théorique. Le système chez Luhmann

Simon Laflamme et Ali Reguigui, Homogénéité et distinction, Sudbury, Prise de parole, 2005. 
est un sujet. En effet, malgré sa tentative d'éliminer le sujet humain de l'analyse, il ne fait que transférer les qualités humaines à son objet d'étude. Le système devient lui-même un sujet agissant et réflexif. Il agit, sélectionne l'information, délimite ses frontières, fait l'autodescription de lui-même, etc. Ensuite, le caractère abstrait de son œuvre, reconnu par tous les commentateurs, n'est en réalité qu'une simple description empirique de nos sociétés. Le niveau d'abstraction se situe plutôt au niveau langagier qu'au niveau scientifique, dans la construction d'un modèle abstrait conformément aux exigences de la science.

Chez Morin, les sujets, bien qu'ils ne constituent qu'un élément dans une longue chaine, sont néanmoins présents. Conformément à sa méthode de la complexité, il refuse de séparer sujet et objet et d'éliminer l'observateur de l'observation. De plus, selon lui, il n'y a pas de sciences sans conscience. Il élabore un nouvel humanisme fondé sur trois qualités : raison, imagination et sens ${ }^{38}$. Enfin, le modèle théorique n'est pas toujours en adéquation avec la description empirique qu'il fait des objets. Peu d'économies de concepts sont à l'œuvre dans sa description du monde social.

Chez Laflamme, il y a une identification à la systémique, mais sans jamais l'assumer pleinement. On peut parler d'affinités, et non d'adhésion. Les concepts systémiques ne sont pas utilisés. De plus, son concept d'échange évacue complètement le sujet. Ensuite, chez Laflamme, la science est sans conscience. La science peut comprendre, par exemple, la souffrance, mais elle ne peut être émue comme ceux qui la vivent. Enfin, chez l'auteur, il y a un souci d'opérationnalisation du modèle, effort absent de chez beaucoup d'auteurs systémiciens, qui oscillent généralement entre soit des applications de la pensée systémique à différents phénomènes, sans souci théorique et sans renouvellement des idées connaissances, soit une forme de "spéculation " sur la systémique, la complexité, sans souci pour les enquêtes. Chez Laflamme, on peut noter particulièrement ce souci de fonder une analyse relationnelle basée sur la preuve. Ses thèses théoriques sont pour la plupart le fruit d'un travail d'opérationnalisation

38 Ali Aï Abdelmalek, "Edgar Morin, sociologue et théoricien de la complexité : des cultures nationales à la civilisation européenne ", Sociétés, vol. 4, n 86, 2004, p. 110 117. 
et d'enquêtes. Les thèses qu'il dirige témoignent également de ce souci d'opérationnalisation de son modèle ${ }^{39}$.

Ces éléments suffisent, à notre avis, à souligner tout l'intérêt pour les systémiciens (cela est vrai pour les autres perspectives) de ne pas considérer leur lorgnette comme homogène si l'on veut espérer un renouvellement de la connaissance. Malgré les limites de ce texte, les raccourcis, les interprétations hâtives des auteurs ou le manque inévitable de nuances, dans ce genre d'exercice, nous espérons attirer l'attention sur l'importance de faire de la relation le véritable terrain de confrontation et de renouvellement de la pensée sociologique.

\section{Conclusion}

Ce texte est animé par un désir : voir la relation devenir un objet central de la sociologie. Mais contrairement à Donati ou à Bajoit, par exemple, nous sommes sceptiques face à toute tentative d'unification du savoir autour d'une notion comme celle de relation ou d'autres catégories comme système ou réseau. Tout d'abord, le souci de démarcation, de positionnement en sociologie empêche toute unification. Par exemple, au lieu de partir de ce qui est acquis en cherchant des solutions aux problèmes théoriques soulevées par une perspective, on a trop tendance à réinventer la roue dans l'indifférence totale des contributions des autres. On peut au moins saluer les tentatives, même vaines, des systémiciens et de quelques sociologues comme Bourdieu d'avoir cherché à intégrer différentes traditions sociologiques et non sociologiques autour d'un seul paradigme. Il faut reconnaittre cependant que ces rares efforts n'ont pas réussi à unifier le savoir. Le souci de différenciation, légitime mais excessif, selon nous, conduit toujours à

Voir Mélanie Girard, Simon Iaflamme et Pascal Roggero, «L'intention est-elle si universelle que le prétendent les théories de l'action ", Nouvelles perspectives en sciences sociales, vol. $1, \mathrm{n}^{\circ} 2,2006$, p. 115-148; Pierre Bouchard, « La théorie de l'action et les parcours de vie", Nouvelles perspectives en sciences sociales, vol. 1, $\mathrm{n}^{\circ} 2,2006, \mathrm{p} .67-114$; Roger Gervais, "La mondialisation : vers une compréhension duelle de l'homogénéisation et de la différenciation ", Nouvelles perspectives en sciences sociales, vol. 2, $n^{\circ}$ 1, 2006, p. 69-100; Paul Jalbert, "Analyse du rôle de l'intention dans les échanges dyadiques ", Nourelles perspectives en sciences sociale, vol. 2, n 1, 2006, p. 101 141. 
faire éclater les catégories autour desquelles certains fondent l'espoir d'unification. Il faut souligner aussi un autre problème nocif à l'unification, celui qui oppose les travailleurs de la preuve aux empiristes et aux théoricistes. Alors que les premiers se donnent comme principe scientifique l'obligation de mener des enquêtes pour soutenir des thèses sur le monde social, d'autres soit se complaisent dans l'empirie sans avancées théoriques, en considérant la connaissance comme allant de soi ; soit se contentent des spéculations sur le monde social hors de toute épreuve empirique ${ }^{4 \prime}$. Le dialogue peut paraître particulièrement fastidieux entre tous ces sociologues et empêche toute avancée dans l'unification d'un savoir autour de la relation.

Pour le moment, si on se fie à notre typologie proposée, nous relevons une pluralité de sociologies relationnelles. Même si tous les auteurs n'insistent pas explicitement sur l'importance de la relation, chaque perspective discutée essaye de résoudre les problèmes majeurs qui divisent les sociologues : agent/structure ; holisme/parties ; individualisme/holisme, etc. En même temps, les trois approches expriment trois manières différentes, mais complémentaires, de faire de l'analyse relationnelle. La typologie relationnelle proposée appuie l'idée que la relation peut-être un objet de confrontation beaucoup plus pertinent que les débats classiques autour de certaines vieilles catégories comme déterminisme, contrainte, intégration, conflit, etc. La relation est première. La sociologie peut réellement fonder une sociologie relationnelle plurielle.

\section{Bibliographie}

Ait Abdelmalek, Ali, "Edgar Morin, sociologue et théoricien de la complexité : des cultures nationales à la civilisation européenne », Sociétés, vol. 4, n 86, 2004, p. $110-117$.

Alexander, Jeffrey, La réduction. Critique de Bourdieu, Paris, Cerf, coll. "Humanités ", 2000 .

Alexander, Jeffrey, Theorical logic in sociology', vol. 1-4, Berkley, University of California Press, 1982-1983.

4" Bernard Lahire, L'esprit sociologique, Paris, Ia Découverte, 2005. 
Archer, Margaret, «Entre la structure et l'action, le temps. Défense du dualisme analytique et la perspective morphogénétique ", Revue du MAUSS, n² 24, 2004, p. $237-349$.

Archer, Margaret, Realist social theory: The morpbogenetic approach, Cambridge, Cambridge University Press, 1995.

Bajoit, Guy, Pour une sociologie relationnelle, Paris, PUF, 1992.

Barel, Yves, Le paradoxe et le systeme, Presses universitaires de Grenoble, 1989.

Berthelot, Jean-Michel, L'intelligence du social. Le pluralisme explicatif en sociologie, Paris, PUF, coll. "Sociologie d'aujourd'hui », 1997.

Bourdieu, Pierre, Questions de sociologie, Paris, Minuit, 1981.

Bouchard, Pierre, "La théorie de l'action et les parcours de vie ", Nonvelles perspectives en sciences sociales, vol. $1, \mathrm{n}^{\circ} 2,2006, \mathrm{p} .67-114$.

Crozier, Michel et Erhard Friedberg, L'acteur et le système, Paris, Seuil, 1977.

Degenne, Alain et Michel Forsé, Les réseaux sociaux, Paris, Armand Colin, 1994.

Donati, Pierpaolo, "Ja relation comme objet spécifique de la sociologie ", Revue du MAUSS, n² 24, 2004, p. 234-254.

Durand, Daniel, La systémique, Paris, PUF, coll. «Que sais-je ? », 1993.

Elias, Norbert, Qu'est-ce que la sociologie?, Paris, Fayard, 1970.

Elias, Norbert, La société des individus, Paris, Fayard, 1991.

Emirbayer, Mustafa, "Manifesto for a Relational Sociology ", American journal of sociology, vol. 103, $\mathrm{n}^{\circ} 2$, septembre, p. 281-317.

Gervais, Roger, "La mondialisation : vers une compréhension duelle de l'homogénéisation et de la différenciation ", Nouvelles perspectives en sciences sociale, vol. $2, \mathrm{n}^{\circ} 1,2006$, p. $60-100$.

Giddens, Anthony, La constitution de la société, Paris, PUF, 1988.

Girard, Mélanie, Simon Laflamme et Pascal Roggero, « L'intention est-elle si universelle que le prétendent les théories de l'action ", Nouvelles perspectives en sciences sociales, vol. $1, \mathrm{n}^{\circ} 2,2006, \mathrm{p} .115-148$.

Godbout, Jacques T. et Alain Caillé, L.'Esprit du don, Paris, La Découverte, coll. « La Découverte poche, Sciences humaines et sociales », 2000.

Goffman, Erving, Asiles: études sur la condition sociale des malades mentaux et autres reclus, Paris, Minuit, 1968.

Goffman, Erving, La mise en scène de la vie quotidienne, Paris, Minuit, 1973.

Goffman, Erving, Les rites d'interaction, Paris, Minuit, 1974.

Jalbert, Paul, "Analyse du rôle de l'intention dans les échanges dyadiques », Nonvelles perspectives en sciences sociales, vol. $2, \mathrm{n}^{\circ} 1,2006, \mathrm{p} .101-141$.

Lahire, Bernard, L'esprit sociologique, Paris, La découverte, 2005.

Laflamme, Simon, Des biens, des idées et des personnes au Canada (1981-1995): un modèle macrosociologique relationnel, Sudbury/Paris, Prise de parole / L'Harmattan, 2000.

Laflamme, Simon, La société intégrée. De la circulation des biens et des idées, New York/ Berne, Peter Iang, Worcester Polytechnic Institute, Studies in Science, Technology and Culture, 1992.

Laflam e, Simon et Reguigui Ali, Homogénéité et distinction, Sudbury, Prise de parole, 2005.

Lapierre, Jean-William, L'analyse des systèmes, Syros, Paris, 1992. 


\section{RACHID BAGAOUI/ UN PARADIGME SYSTEMIQUE RELATIONNEL...}

Lazega, Emmanuel, Réseanx sociaux et structures relationnelles, Paris, PUF, coll. «Que saisje?", PUF, 1998.

Lćvi-Strauss, Claude, Anthropologie structurale, Paris, Plon, 1958.

Lévis-Strauss, Claude, Les structures élémentaires de la parenté, Paris, Mouton, Maison des sciences de l'homme, coll. «De rééditions », 1967.

Lugan, Jean-Claude (dir.), Lexique systémique et prospective, Toulouse, Conseil économique et social de la région Midi-Pyrénées, 2006.

Iugan, Jean-Claude, La systémique sociale, Paris, PUF, coll. "Que-sais-je?», 1995.

Luhmann, Niklas, "Développements récents en théorie des systèmes ", dans Gérard Duprat (dir.), Connaissance du politique, Paris, PUF, 1990, p. 281-293.

Luhmann, Niklas, Ecological communication, Chicago, University of Chicago Press, 1989.

Luhmann, Niklas, Social systems, Standford, Stanford University Press, 1995.

Mauss, Marcel, Sociologie et anthropologie, Paris, PUF, coll. «Sociologie aujourd'hui ", 1973.

Morin, Edgar, Le cinéma ou l'bomme imaginaire, Paris, Éditions De Minuit, 1985.

Morin, Edgar, Le défz du XXle siècle. Relier les connaissances, Paris, Seuil, 1999.

Morin, Edgar, L'Homme et la mort, Paris, Buchet/Chastel, 1951.

Morin, Edgar, Introduction à la pensée complexe, Paris, ESF, 1992.

Morin, Edgar, Penser l'Europe, Paris, Gallimard, 1987.

Morin, Edgar, La tête bien faite. Repenser la réforme. Réformer la pensée, Paris, Seuil, 1999.

Morin, Edgar et Jean-J couis I.e Moigne, L'intelligence de la complexité, Paris, L'Harmattan, 1999.

Parsons, Talcott, Théorie générale des systèmes, Paris, Dunod, 1973.

Revue du MAUSS, Une theirie sociologique générale est-elle pensable?, $\mathrm{n}^{\circ} 24,2004$.

Rosnay, Joël de, Le macroscope. I'ers une vision globale, Paris, Seuil, coll. « Points ", 1975.

Touraine, Alain, Pour la sociologie, Paris, Seuil, 1974.

Touraine, Alain, La voix et le regard, Paris, Seuil, 1978.

Von Bertalanffy, Ludwing, Théorie générale des systèmes, Paris, Dunod, 1990.

Wasserman, Stanley et Katherine Faust, Social network analysis: Methods and applications, New York, Cambridge University Press, 1994.

Watzlawick, Paul, Janet $\mathrm{H}$. Beavin et Don D. Jackson, Une logique de la communication, Paris, Seuil, 1972. 